\title{
Bestimmung der Temperaturverteilung im Kraftwerkskessel mit einem Gray-Box-Modell als Basis für die Rauchgas-Entstickung mittels SNCR-Verfahren
}

\author{
Christian Neu und Dirk Kiehn
}

STEAG Power Saar GmbH, Saarbrücken, Trierer Straße 4

Andreas Schütze

Universität des Saarlandes, Lehrstuhl für Messtechnik, Saarbrücken, Campus A5.1

\section{Zusammenfassung}

Eines der großtechnischen Verfahren zur gesetzlich geforderten Entstickung der Rauchgase von Steinkohlekraftwerken ist das SNCR-Verfahren (Selective Non Catalytic Reduction), bei dem die verwendeten Reduktionsmittel unmittelbar in die heißen Rauchgase eingedüst werden. Die mit diesem Verfahren erreichbare Stickoxid-Abscheidung hängt stark vom Sauerstoffgehalt und der Rauchgastemperatur ab (s. Bild 1) und fand in Großkraftwerken keine weite Verbreitung, weil die räumliche Verteilung der Rauchgastemperatur unter den dynamischen Randbedingungen des Kesselbetriebes online nicht genau genug gemessen werden kann. Mit einem Gray-Box-Modell werden einem CFD-System durch Messung und Abschätzung Startwerte zur Verfügung gestellt, mit denen die Berechnung der Temperatur in finiten Volumenelementen (kurz: FV) mit der Kantenlänge 1 Meter im Feuerraum des Dampferzeugers unter Betriebsbedingungen quasi-online, im vorliegenden Fall alle 20 Sekunden, ermöglicht wird.

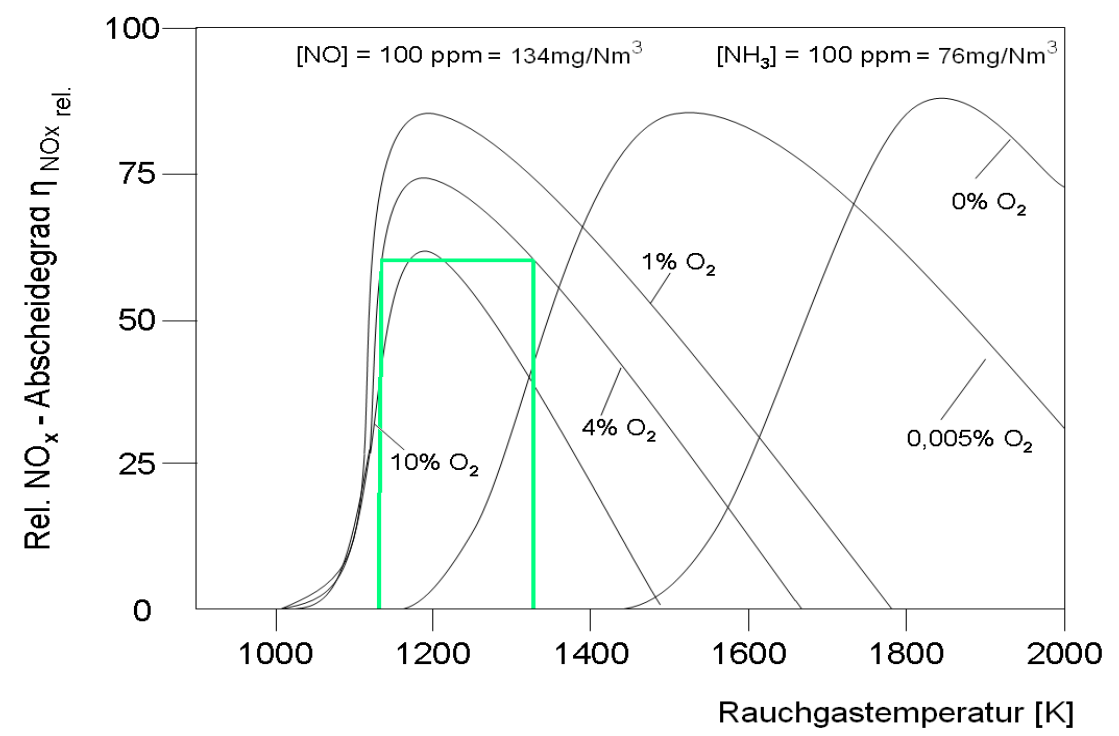

Bild 1 Temperaturabhängigkeit des Abscheidegrades bei verschiedenen Sauerstoffanteilen in der Umgebungsatmosphäre; günstiges Temperaturfenster bei betriebsüblichen $4 \%$ Sauerstoffgehalt $\mathrm{rd} .900{ }^{\circ} \mathrm{C}$ bis $1100{ }^{\circ} \mathrm{C}$ [1].

\section{Einleitung}

Durch ein abbaubedingtes Erdbeben und einen nachfolgend verhängten Abbaustopp wurde am Kraftwerksstandort Fenne eine kurzfristige Umstellung der Brennstoffbasis notwendig. Die im Kraftwerksblock Modellkraftwerk Völklingen (kurz: MKV) bis dahin praktizierte Primär-Entstickung durch gestufte Zuführung der Verbrennungsluft reichte zur Einhaltung des genehmigten Grenzwertes für Stickoxide nicht 
mehr aus, so dass eine Sekundär-Entstickung notwendig wurde. Da die Investitionskosten für eine katalytische SCR-Anlage unwirtschaftlich hoch waren, entschied man sich für das weit kostengünstigere SNCR-Verfahren, obwohl Zweifel an der erreichbaren Entstickungsqualität aufgrund des nicht gelösten Problems der unbekannten Temperaturverteilung herrschten.

Einen Lösungsansatz bietet die Berechnung der Temperaturverteilung durch ein CFD-System. Hierzu müssen allerdings Möglichkeiten gefunden werden, die über jeden einzelnen Brenner eingetragene Wärmemenge lokal zu bestimmen und somit Startwerte für das CFD-System zu setzen. Die Startwerte selbst müssen im Sinne einer online-Kalibrierung an die jeweils herrschenden Betriebs- und Feuerungsbedingungen, wie Kohleschieflagen und Kohlequalitäten, angepasst werden. Diese sind mit den gängigen Verfahren für die beabsichtigte Anwendung nicht schnell genug messbar und müssen alternativ bestimmt werden. Zum Teil werden sie von gemessen Größen abgeleitet. Gleiches gilt für die Turbulenzverhältnisse in der Rauchgasströmung und die Behinderung des Wärmeüberganges vom Rauchgas auf das Speisewasser des Kessels durch lokale Aschebelegung, das sogenannte Fouling.

\section{Struktur des Gray-Box-Modells}

Mit der in Bild 2 gewählten Struktur ist es möglich, die Fehler bei der Bestimmung der nicht-messbaren Eingangs- und Berechnungsparameter des CFD-Systems zu korrigieren und somit an wechselnde Betriebsbedingungen anzupassen. Durch Vergleich von vier mittels PT100 gemessenen Rauchgastemperaturen am Ende des modellierten Kesselbereiches (Höhe 55 Meter) mit den lokal korrespondierenden Modelltemperaturen, können die für eine Iteration wesentlichen CFD-Parameter wie die Flammentemperatur, der Fouling-Faktor und der die Turbulenzverhältnisse beschreibende Quotient $\kappa / \varepsilon$ angepasst werden. Dies geschieht mittels eines künstlichen neuronalen Netzes (KNN), mit dem diese sogenannten dynamischen Skalierungsfaktoren in geeigneter Weise verändert werden. Dabei werden die Skalierungsfaktoren mit unterschiedlichen Lernraten versehen, um dem stark unterschiedlichen Zeitgang der einzelnen Prozesse Rechnung zu tragen.

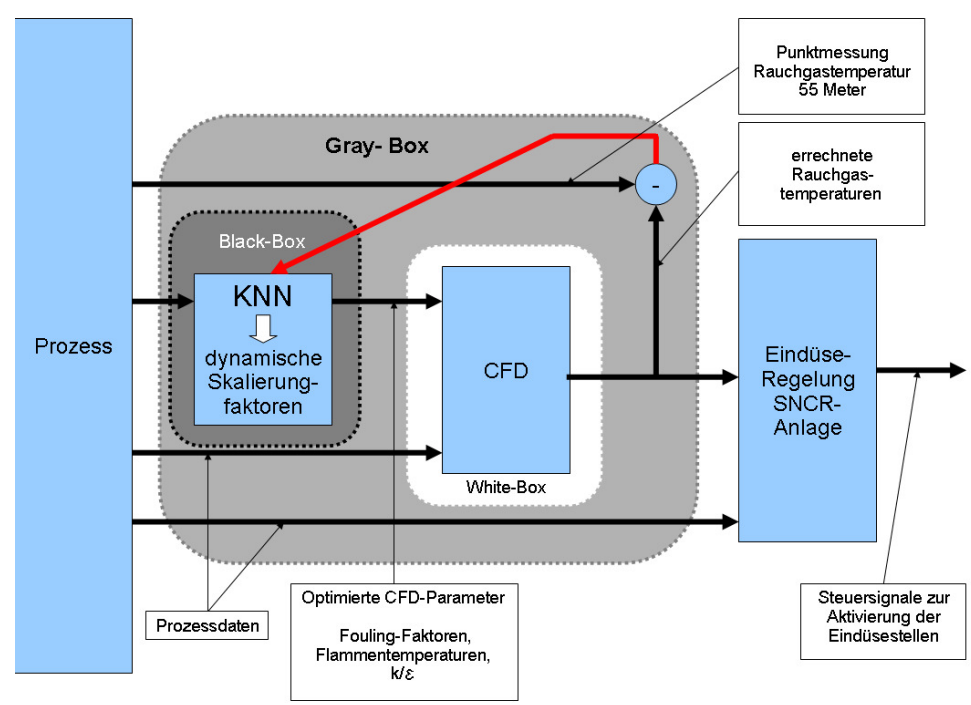

Bild 2 Strukturskizze des Gray-Box-Modells zur Berechnung der Temperaturverteilung im Dampferzeuger

\section{Modellierung der nicht-messbaren Betriebsgrößen}

\subsection{Kohleschieflage}

Der Kohlestaubstrom - und dementsprechend der Wärmeeintrag - verteilt sich nach dem Mahlprozess nicht gleichmäßig auf die nachgeschalteten Brenner. Zur Abschätzung dieser nicht vermeidbaren Schieflage dienen CMOS-Kameras, die in den Kesselwänden in Brennernähe zur Flammenbeobachtung angeordnet sind (s. Bild 3). 


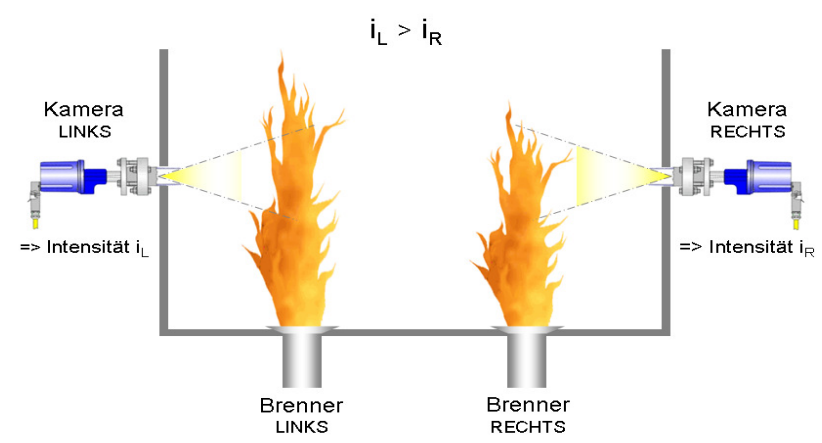

Bild 3 Anordnung der CMOS-Kameras zur Abschätzung der Kohlestaubschieflage, hier $i_{L}>i_{R}$

Durch Vergleich der mit den CMOS-Kameras gemessenen Strahlungsintensitäten der Einzelbrenner wird mittels der gemessenen Gesamtkohlemenge auf die Schieflage des Kohlemassenstromes rückgeschlossen und so die im Einzelbrenner umgesetzte Kohlemenge berechnet.

\subsection{Bestimmung der Flammentemperatur}

Wenn die Kohlemenge, die über die einzelnen Brenner eingetragen wird bekannt ist, kann nach Bestimmung der Flammentemperatur die jeweilige lokal eingebrachte Wärmemenge bestimmt werden. Die Flammentemperatur wird dazu mittels eines jeweils pro Brenner installierten Strahlungsthermometers abgeschätzt (s. Bild 4). Dieses besteht aus einer luftumspülten schwarzen Kugel, deren Temperatur mit einem Mantelwiderstandsthermometer gemessen wird. Mittels Strahlungspyrometer werden die einzelnen Strahlungsthermometer auf die Flammentemperatur kalibriert. Zwischen der Flammentemperatur und der Temperatur des Strahlungsthermometers besteht ein nahezu proportionaler Zusammenhang. In den entsprechenden Proportionalitätsfaktor fließen allerdings die betrieblich unvermeidbaren Schwankungen des Verbrennungsprozesses ein, die u.a. von wechselnden Heizwerten der Einsatzkohle und den Verbrennungsluftverhältnissen herrühren. Um dies zu berücksichtigen, wird der Faktor über das KNN variiert und es handelt sich somit um einen der dynamischen Skalierungsfaktoren (s. Abschnitt 2)
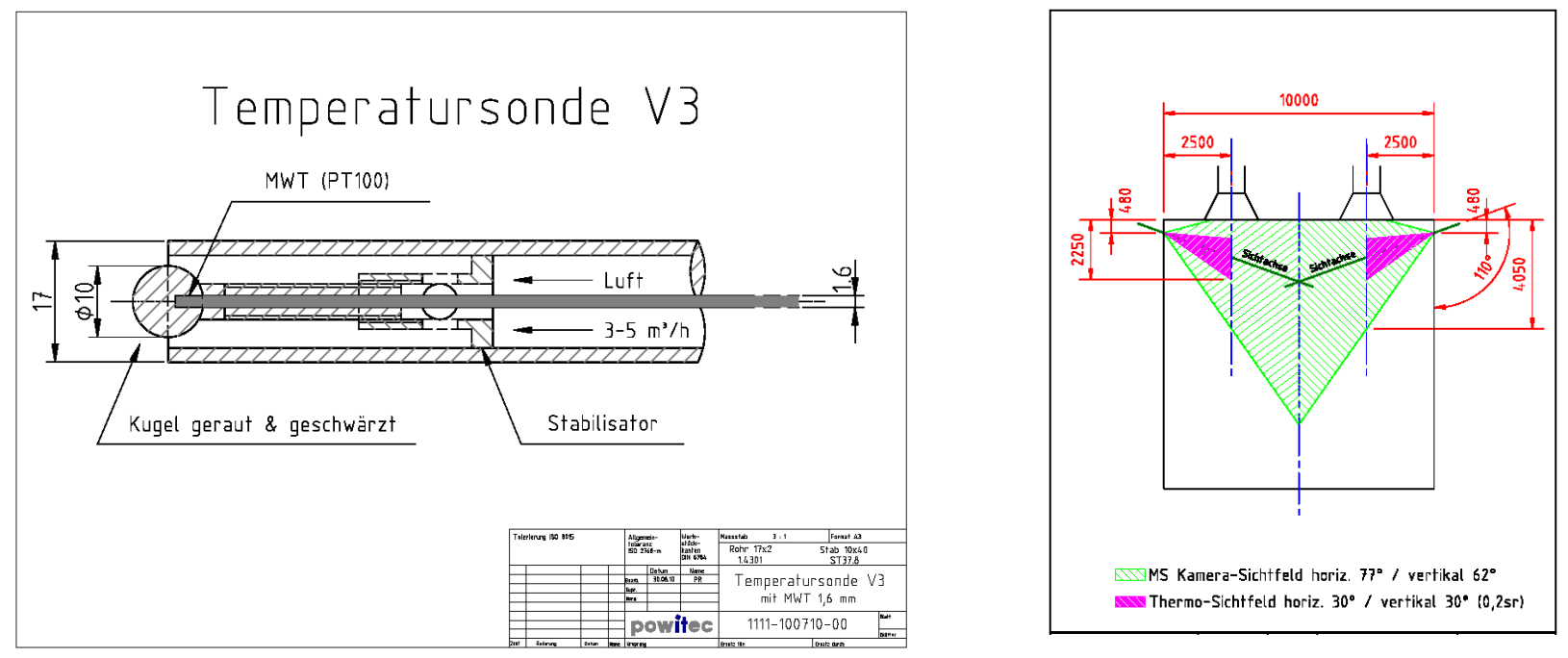

Bild 4 Schnittbild und Anordnung der Strahlungsthermometer im Dampferzeuger [2]

\subsection{Kesselverschmutzung - Fouling}

Der Wärmeübergang auf das in den Kesselrohren fließende Speisewasser, und dementsprechend die im Rauchgas verbleibende Wärmemenge, wird durch Aschebelag sehr stark beeinflusst und muss bei der Temperaturberechnung berücksichtigt werden. Dazu wird mit dem Fouling-Faktor ein weiterer dynamischer 
Skalierungsfaktor eingeführt, mit dem der Einfluss des Aschebelages auf den Übergang von Strahlungswärme auf die Kesselrohre und das darin strömende Speisewasser modelliert wird.

\subsection{Turbulenzmodell}

Der konvektive Wärmeübergang vom Rauchgas auf die Kesselrohre und letztlich auf das durchströmende Speisewasser hängt stark von den Turbulenzverhältnissen der Rauchgasströmung ab. Das angewandte CFDSystem basiert auf dem $\kappa / \varepsilon$-Modell, wobei der Quotient vereinfacht die Umwandlung von kinetischer Energie in Wärme repräsentiert. Der Quotient ist im Gray-Box-Modell ebenfalls ein dynamischer Skalierungsfaktor, mit dem die Änderung der Turbulenzverhältnisse bei unterschiedlichen Lasten modelliert werden kann.

\section{Weitere Modellierungsaspekte}

Eine im modellierten Kesselbereich befindliche Berührungsheizfläche des Überhitzers wird über die Enthalpiebilanz des Dampfes bzgl. ihrer Wärmentnahme aus dem Rauchgas bewertet. Auch der Wärmeentzug durch die Verdampfung des mit dem Reagenzmittel eingedüsten Trägerwassers wird berücksichtigt. Ebenso wird die zur Nachverbrennung unverbrannter Rauchgasbestandteile in den Feuerraum eingeblasene Oberluft thermisch bilanziert.

\section{Fazit und Bewertung}

Durch die Definition eines geeigneten Kriteriums kann die Reagenzeindüsung auf Basis der berechneten Temperaturverteilung angesteuert werden. Mit diesem Konzept wird im Kraftwerksblock MKV der genehmigte Emissionsgrenzwert über alle Lastbereiche eingehalten, dies auch unter den anspruchsvollen dynamischen Anforderungen bei der Darstellung von Regelenergie. In Bild 5 ist je eine Momentaufnahme der Temperaturverteilung im Dampferzeuger für zwei Lastfälle nach einem Rechnungslauf beispielhaft dargestellt.
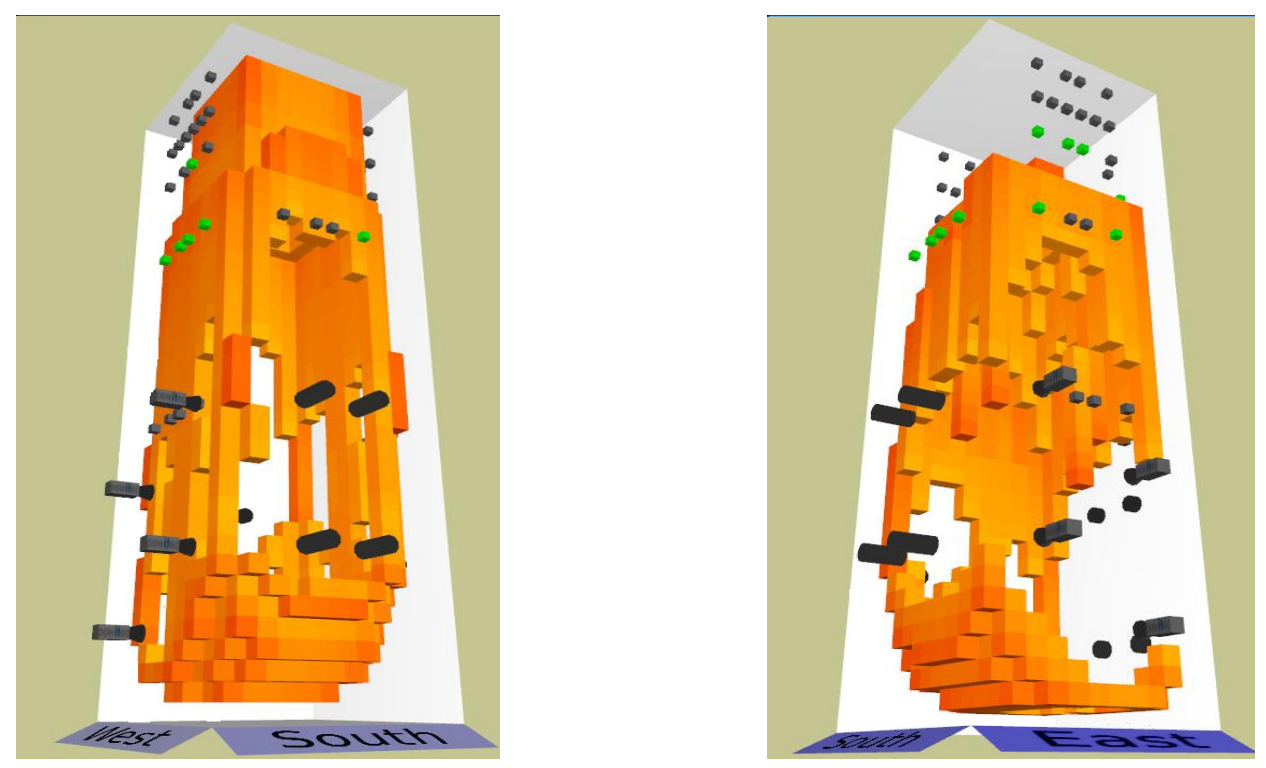

Bild 5 FV mit Rauchgastemperatur zw. $900{ }^{\circ} \mathrm{C}$ und $1100{ }^{\circ} \mathrm{C}$ bei Kessel-Volllast (links) und Kessel-Teillast (rechts)

\section{Literatur}

[1] Bernd v. d. Heide: Abfallwirtschaft- und Energiekonferenz in Berlin 2008; Band 4

[2] F.Wintrich, P.Richter: Entwicklungs- und Anordnungszeichnung Fa. Powitec, Essen 